$\mathrm{CDF} / \mathrm{PUB} / \mathrm{BOTTOM} / \mathrm{PUBLIC} / 5200$

FERMILAB-Conf-00/007-E

Version 2.0

November 19, 2018

\title{
Brief Report from the Tevatron
}

\author{
Manfred Paulini \\ Lawrence Berkeley National Laboratory \\ Berkeley, California 94720, USA \\ (Representing the CDF and DØ Collaborations)
}

\begin{abstract}
We report on the $B$ physics prospects from the Fermilab Tevatron, summarizing the $B$ physics goals of the $\mathrm{CDF}$ and $\mathrm{D} \varnothing$ experiments using their upgraded detectors. We discuss the time schedule for completion of the detector upgrades and summarize the current measurement of the $C P$ violation parameter $\sin 2 \beta$ at $\mathrm{CDF}$.
\end{abstract}

Plenary talk presented at XIX International Symposium on Lepton and Photon Interactions at High Energies Stanford University, August 9-14, 1999. 



\section{Brief Report from the Tevatron}

Manfred PauliniPaulini, Manfred

Lawrence Berkeley National Laboratory

Berkeley, California 94720

\section{Introduction}

It might appear surprising to include a report from the Fermilab Tevatron, a protonantiproton collider, in a session about "Brief Reports from the $B$ factories". Does this mean the Tevatron would qualify as a $B$ factory? There are two advantages of studying $B$ physics at the Tevatron. First, all $B$ hadrons are produced; not only charged and neutral $B$ mesons as at the $B$ factories, but also $B_{\mathrm{S}}^{0}$ mesons and $b$ baryons. The second advantage is the $b$ quark production cross section, which is about $1 \mathrm{nb}$ at the $\Upsilon(4 S)$ resonance while it is about $50 \mu \mathrm{b}$ for $p \bar{p}$ collisions at $\sqrt{s}=$ $1.8 \mathrm{TeV}$. This is an enormous cross section which is about 50,000 times larger at the Tevatron than at the $B$ factories. It resulted in about $5 \times 10^{9} b \bar{b}$ quark pairs being produced during the 1992-1995 data taking period of the Tevatron, called Run I. To illustrate the enormous $b$ production rate at the Tevatron, we compare the yield of fully reconstructed $B$ mesons between the CLEO experiment and CDF. In a data sample of about $3000 \mathrm{pb}^{-1}$, CLEO reconstructs about $200 B$ mesons decaying into $J / \psi K^{+}$[1] while CDF finds in a sample of about $100 \mathrm{pb}^{-1}$ of data a signal of about $1000 \mathrm{~J} / \psi \mathrm{K}^{+}$events with a good signal-to-background ratio [2].

The goal of the $B$ factories is to discover $C P$ violation in $B^{0} \rightarrow J / \psi K_{S}^{0}$ decays. CDF has already presented a measurement of $C P$ violation in the $B$ meson system [3], measuring the time-dependent asymmetry in the yield of $J / \psi K_{S}^{0}$ events coming from a $B^{0}$ versus $\bar{B}^{0}$ :

$$
\mathcal{A}_{C P}(t) \equiv \frac{N\left(\bar{B}^{0}(t)\right)-N\left(B^{0}(t)\right)}{N\left(\bar{B}^{0}(t)\right)+N\left(B^{0}(t)\right)}=\sin 2 \beta \sin \Delta m_{d} t .
$$

This asymmetry is directly related to the $C P$ violation parameter $\sin 2 \beta$.

\subsection{CDF Measurement of $\sin 2 \beta$}

Here, we briefly summarize CDF's initial measurement of $\sin 2 \beta$. Figure 1 (a) shows the $J / \psi K_{S}^{0}$ yield at CDF, where $395 \pm 31$ events have been identified. This is currently the world's largest sample of fully reconstructed $J / \psi K_{S}^{0}$ events. Measuring a 

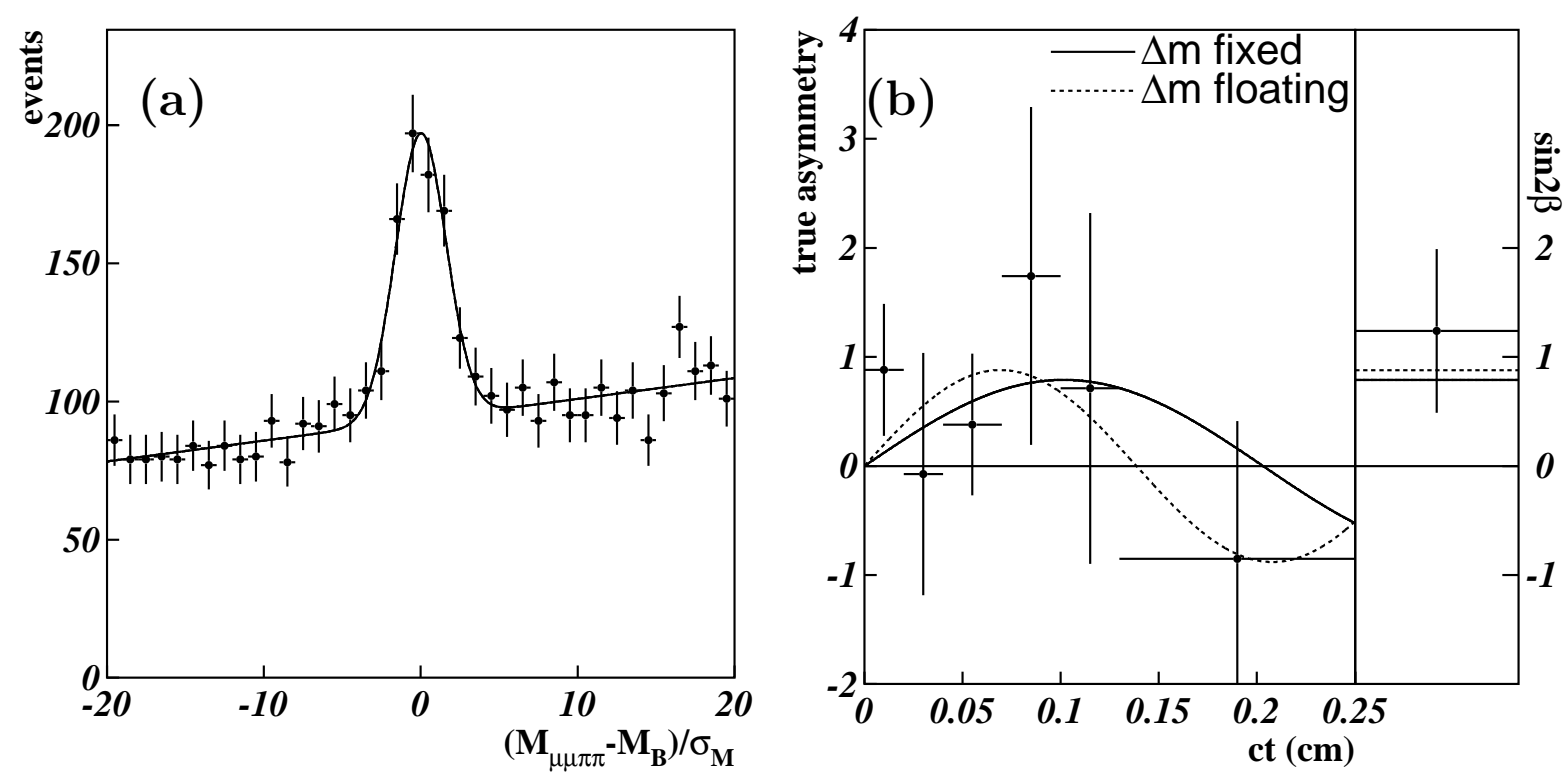

Figure 1: (a) Normalized mass distribution of $J / \psi K_{S}^{0}$ candidates. (b) True asymmetry $\sin 2 \beta$ as a function of the reconstructed $J / \psi K_{S}^{0}$ proper decay length. The events with low decay length resolution are shown separately on the right.

$C P$ asymmetry requires knowing whether the $J / \psi K_{S}^{0}$ originated from a $B^{0}$ or $\bar{B}^{0}$ meson. This is usually referred to as $B$ flavor tagging. Several methods of $B$ flavor tagging exist. Some of them exploit the other $B$ hadron in the event and search for a lepton from the semileptonic decay of the other $B$ hadron or determine the net charge of the jet produced by the other $b$ quark. These two methods are called lepton tagging and jet charge tagging, respectively. The $B$ flavor can also be determined by searching for pions which are produced through fragmentation or $B^{* *}$ mesons in correlation with the $B$ meson of interest. This method is known as same side tagging.

$B$ flavor tagging is the crucial element for a $C P$ violation measurement at the Tevatron. The figure of merit quantifying how well a flavor tagging algorithm works is the so-called effective tagging efficiency $\varepsilon \mathcal{D}^{2}$. Here, $\varepsilon$ is the efficiency for obtaining a particular flavor tag, and $\mathcal{D}$ is the dilution defined by the number of right tags $\left(N_{R}\right)$ and the number of wrong tags $\left(N_{W}\right): \mathcal{D}=\left(N_{R}-N_{W}\right) /\left(N_{R}+N_{W}\right)$. CDF determined the tagging power of various tagging methods with data measuring the time dependence of $B^{0} \bar{B}^{0}$ flavor oscillations. Such a measurement serves as a demonstration that a particular flavor tag does work in a hadron collider environment and determines its $\varepsilon \mathcal{D}^{2}$. Figure 2(a) shows the measured mixing asymmetries as a function of proper decay length using a same side tag [2, 国. From this measurement, CDF extracts 

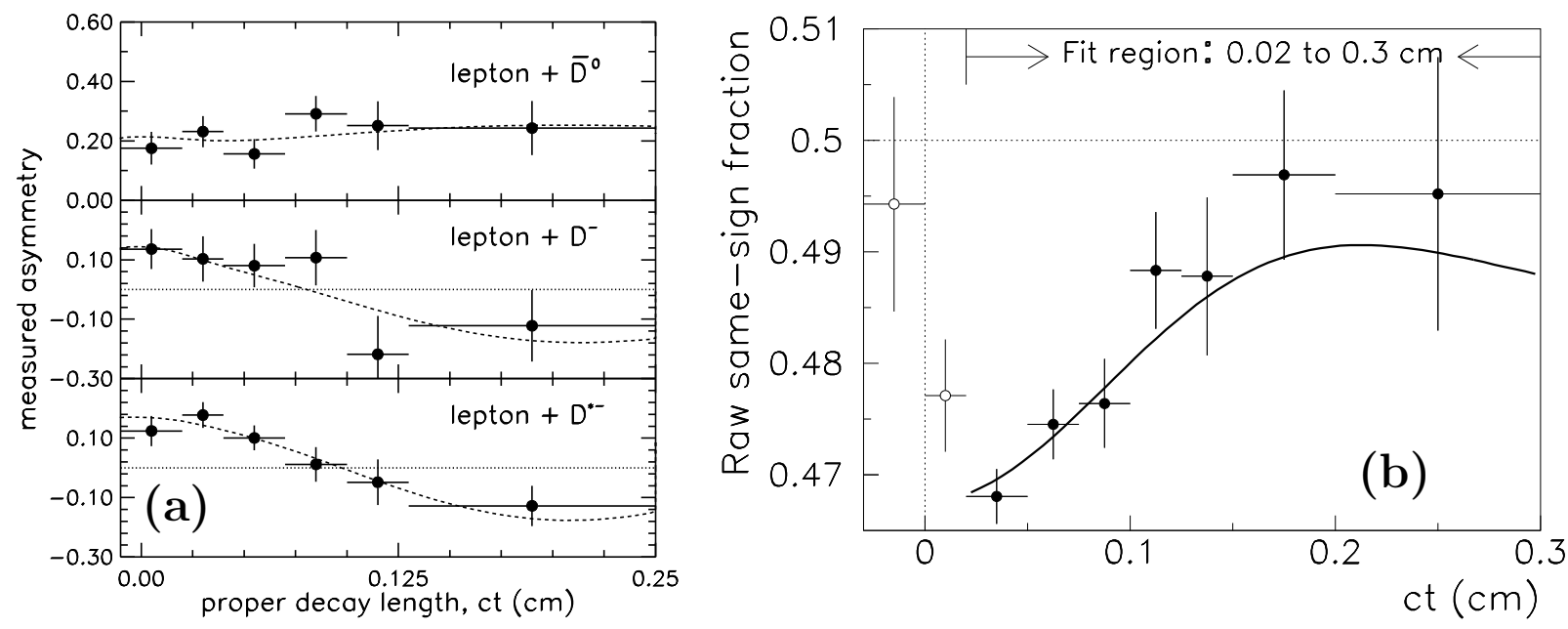

Figure 2: (a) Measured asymmetries as a function of proper decay length using a same side tag for the decay signatures: $\bar{D}^{0} \ell^{+}$(top), $D^{-} \ell^{+}$(middle), and $D^{*-} \ell^{+}$(bottom). (b) Fraction of mixed events as a function of proper decay length using a jet charge and lepton flavor tag.

$\Delta m_{d}=\left(0.471_{-0.068}^{+0.078} \pm 0.034\right) \mathrm{ps}^{-1}$ and the effective tagging efficiency for the same side tag to be $\varepsilon \mathcal{D}^{2}=(1.8 \pm 0.4 \pm 0.3) \%$. As another example, Fig. 2(b) shows the fraction of mixed events as a function of proper decay length using a jet charge and lepton flavor tag [5]. This measurement yields $\Delta m_{d}=(0.500 \pm 0.052 \pm 0.043) \mathrm{ps}^{-1}$ as well as $\varepsilon \mathcal{D}^{2}=(0.91 \pm 0.10 \pm 0.11) \%$ and $\varepsilon \mathcal{D}^{2}=(0.78 \pm 0.12 \pm 0.08) \%$ for a lepton tag and jet charge tag, respectively.

Applying all three tagging methods to CDF's sample of $J / \psi K_{S}^{0}$ events gives the $C P$ asymmetry distribution shown in Fig. 1(b). The data prefer a positive asymmetry, resulting in a measurement of $\sin 2 \beta=0.79 \pm 0.39 \pm 0.16$. This can be translated into a limit on $\sin 2 \beta$ being positive $(0<\sin 2 \beta<1)$ at $93 \%$ confidence level. This is the best direct measurement of $C P$ violation in the $B$ system to date. With this result, CDF demonstrated that a $C P$ violation measurement is feasible at the Tevatron in Run II. Returning to our initial question, we think the Tevatron does qualify as a $B$ factory capable of measuring $C P$ violation. 

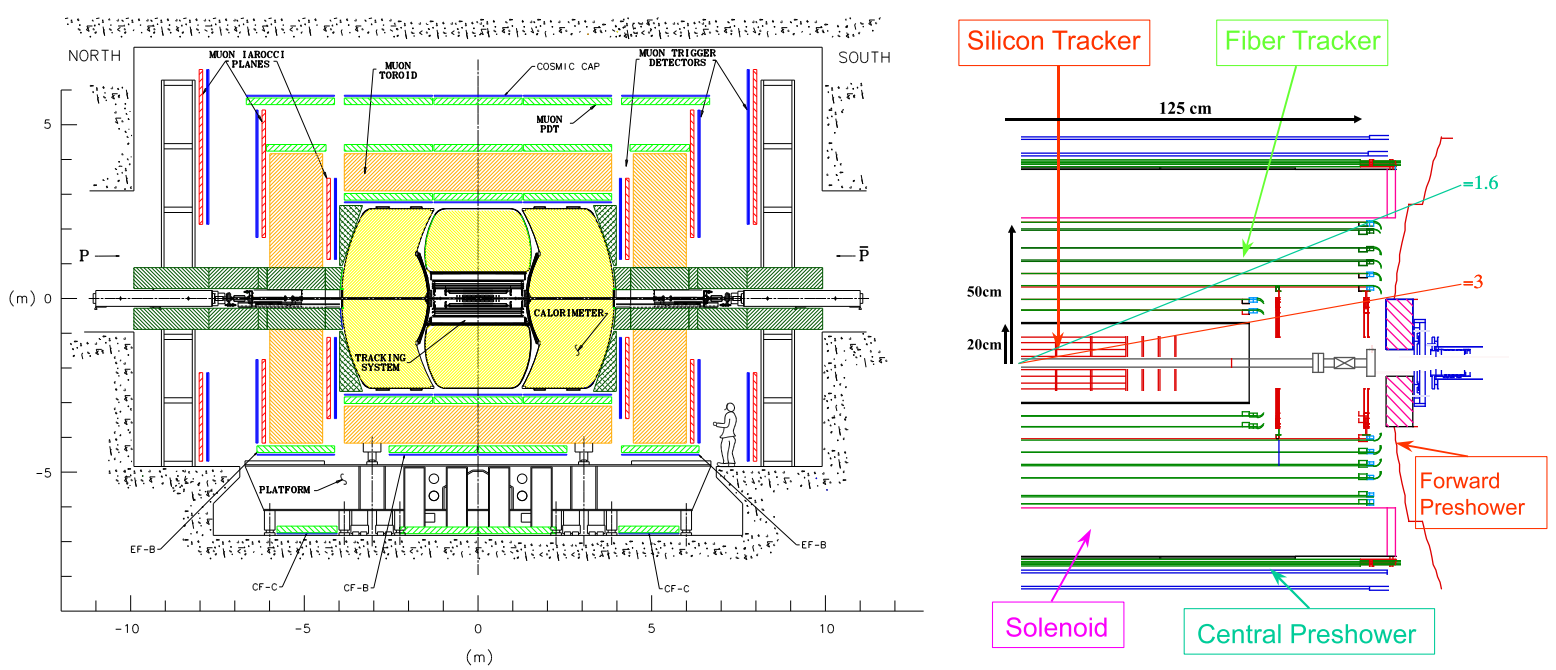

Figure 3: Left: Cross section of the upgraded D $\varnothing$ detector. Right: Longitudinal view of the $\mathrm{D} \varnothing$ tracking system.

\section{The Tevatron in Run II}

The most important element of the Tevatron upgrade for Run II is the Main Injector. It is a new $150 \mathrm{GeV}$ accelerator, half the circumference of the Tevatron, which will increase the antiproton intensity into the Tevatron, providing 20 times higher luminosities. The Main Injector project was finished in June 1999 with the first beam circulating at that time.

Run II of the Tevatron had originally been defined as $2 \mathrm{fb}^{-1}$ being delivered in two years to the collider experiments. Run II has recently been extended beyond the initial two years, to continue until 2006 with no major shutdowns, maximizing the delivered luminosity to a total of up to $15 \mathrm{fb}^{-1}$. The current Fermilab schedule fixes the start of Run II in March 2001.

\subsection{DØ Detector Upgrade}

The $\mathrm{D} \varnothing$ detector upgrade is built on previous strengths, combining excellent calorimetry with good muon coverage and purity. A cross section of the upgraded D $\varnothing$ detector is shown in Fig. 3. The most important improvement is a superconducting solenoid ( $B=2 \mathrm{~T}$ ) providing significantly improved tracking capabilities (see right hand side of Fig. 3). A central fiber tracker consisting of eight superlayers of scintillating fibers allows a measurement of the charged particle momentum. Together with the silicon 

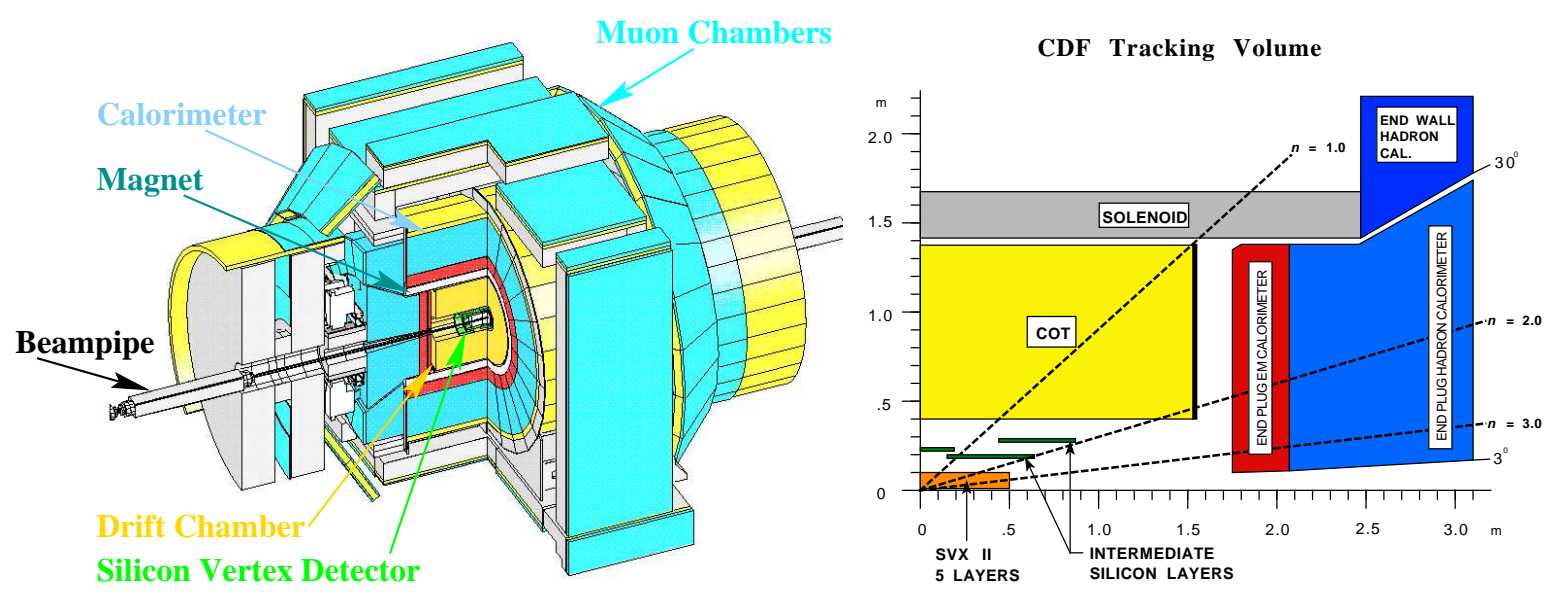

Figure 4: Left: Schematic cut-away view of the CDF II detector. Right: Longitudinal view of the upgraded CDF tracking system.

micro-strip tracker, a momentum resolution of $\sigma\left(p_{T}\right) / p_{T}=0.002 p_{T}$ will be achieved. The silicon tracker consists of six barrels with four layers each ( $r \phi$ and $r z$ readout) and $12+4$ forward disks reaching out to $1.25 \mathrm{~m}$ in $z$. In addition, improvements to the muon system will allow enhanced muon triggering for $p_{T}>1.5 \mathrm{GeV} / c(|\eta|<2)$. Central and forward preshower detectors will improve electron identification and triggering on electrons with $p_{T}>1 \mathrm{GeV} / c(|\eta|<2.5)$. Finally, an impact parameter trigger detecting tracks from displaced vertices is under development.

The current D $\varnothing$ schedule expects the central and forward preshower fabrication as well as the central fiber tracker project to be completed by June 2000. The silicon tracker will be finished by September 2000 and the full tracking system installed and hooked up a few weeks later. The muon system will be in place by November 2000 and the calorimeter electronics by January 2001, allowing DØ to be rolled in and ready for beam by February 2001.

\subsection{CDF Detector Upgrade}

The goal for the CDF detector upgrade is to maintain detector occupancies at Run I levels, although many of the detector changes also provide qualitatively improved detector capabilities. A schematic view of the CDF II detector is shown in Fig. 因. One major improvement is to the charged particle tracking system (see Fig. (1), vital for the $B$ physics program at $\mathrm{CDF}$. A new silicon vertex detector will consist of five layers of double sided silicon from radii of $2.9 \mathrm{~cm}$ to $10 \mathrm{~cm}$. The silicon detector will include three modules covering the entire $p \bar{p}$ luminous region. In addition, an 


\begin{tabular}{l|cc}
\hline Flavor tag & $\varepsilon \mathcal{D}^{2}$ Run I & $\varepsilon \mathcal{D}^{2}$ Run II \\
\hline \hline Same side tag & $(1.8 \pm 0.4 \pm 0.3) \%$ & $2.0 \%$ \\
Jet charge tag & $(0.78 \pm 0.12 \pm 0.08) \%$ & $3.0 \%$ \\
Lepton tag & $(0.91 \pm 0.10 \pm 0.11) \%$ & $1.7 \%$ \\
Kaon tag & - & $2.4 \%$ \\
\hline
\end{tabular}

Table 1: Summary of effective $B$ flavor tagging efficiencies $\varepsilon \mathcal{D}^{2}$ measured at $\mathrm{CDF}$ in Run I and corresponding projections for Run II.

intermediate silicon layer consisting of two double-sided silicon sensors at larger radii permits stand-alone silicon tracking out to $|\eta|=2$. A new open cell drift chamber (COT) will operate at a beam crossing time of $132 \mathrm{~ns}$ with a maximum drift time of $\sim 100$ ns. The COT consists of 96 layers arranged in four axial and four stereo superlayers. It also provides $\mathrm{d} E / \mathrm{d} x$ information for particle identification.

The upgrades to the muon system almost double the central muon coverage. A new scintillating tile plug calorimeter will give good electron identification up to $|\eta|=2$. New front-end electronics will be installed, and a DAQ upgrade will allow the operation of a pipelined trigger system. Finally, two additional upgrade projects significantly enhancing the $B$ physics capabilities of the CDF II detector have been approved. These include the installation of a low-mass radiation hard single-sided silicon detector with axial strips at very small radius of $\sim 1.6 \mathrm{~cm}$, as well as the installation of a time-of-flight system employing 216 three-meter-long scintillator bars located between the outer radius of the COT and the superconducting solenoid.

The current $\mathrm{CDF}$ schedule foresees cosmic ray running of the detector at the beginning of 2000 and expects the COT to be installed in April 2000. A commissioning run will take place from August to November of 2000. The silicon upgrade will be complete by September 2000 and installed by January 2001. The full CDF II detector will be ready for collisions by March 2001 .

\section{Run II $B$ Physics Prospects}

When discussing the Run II $B$ physics prospects in this section, we will refer to a data sample of $2 \mathrm{fb}^{-1}$ delivered in two years. We will focus on the prospects for the $\mathrm{CDF}$ experiment. $\mathrm{D} \emptyset$ has similar expectations.

For a measurement of $\sin 2 \beta$, CDF expects $10,000 J / \psi K_{S}^{0}$ events in $2 \mathrm{fb}^{-1}$ with the $J / \psi$ decaying to muon pairs and $K_{S}^{0} \rightarrow \pi^{+} \pi^{-}$. With the enhanced tracking and vertexing capabilities, extended lepton coverage and better particle identification, $\mathrm{CDF}$ will improve the effective flavor tagging efficiencies for the different taggers as 


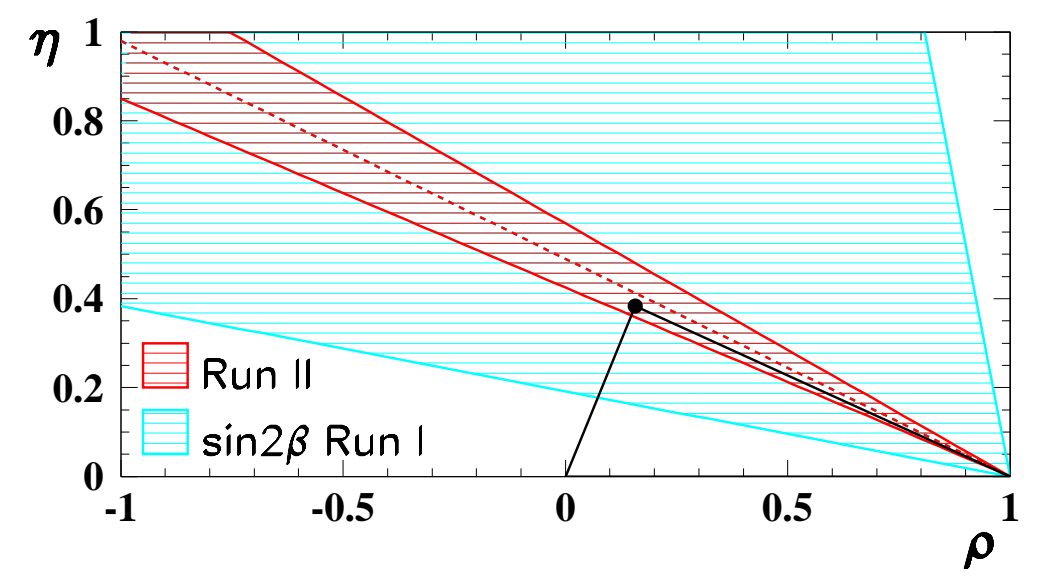

Figure 5: Illustration of the current CDF result on $\sin 2 \beta$ in the $(\rho, \eta)$-plane (light shaded area) and expected improvement in Run II (dark shaded area).

detailed in Table 1 to a total $\varepsilon \mathcal{D}^{2}$ of approximately $9.1 \%$. With this, CDF expects to measure $\sin 2 \beta$ with an uncertainty of $\sim 0.07$. Figure 5 shows the current CDF result on $\sin 2 \beta$ in the $(\rho, \eta)$-plane where the light shaded area indicates the present $1 \sigma$ uncertainty. To illustrate the improvements in Run II, the dark shaded area displays the expected error on $\sin 2 \beta$ in Run II with $2 \mathrm{fb}^{-1}$.

With respect to other $C P$ modes, $\mathrm{CDF}$ plans to measure the time dependence of the $C P$ asymmetry in $B^{0} \rightarrow \pi^{+} \pi^{-}$decays, determining $\sin 2 \alpha$. CDF will use a displaced track trigger which will trigger on hadronic tracks from long-lived particles such as $B$ hadrons. With a fast track trigger at Level 1 , CDF finds track pairs in the COT with $p_{T}$ greater than $1.5 \mathrm{GeV} / c$. At Level 2, these tracks are linked into the silicon vertex detector, and cuts on the track impact parameter $d>100 \mu \mathrm{m}$ $(\sigma(d) \sim 25 \mu \mathrm{m})$ are applied. CDF expects to collect 4000-7000 $B \rightarrow \pi \pi$ events, assuming $\mathcal{B}\left(B^{0} \rightarrow \pi^{+} \pi^{-}\right)=\left(4.7_{-1.5}^{+1.8} \pm 0.6\right) \cdot 10^{-6}$ as measured by CLEO [6]. In addition, there will be about four times more $B \rightarrow K \pi$ decays. The implications of this background for an extraction of $\sin 2 \alpha$ at $\mathrm{CDF}$ are still under study.

For a measurement of $\sin \gamma$ in Run II, CDF can use $B_{\mathrm{S}}^{0}$ mesons. A signal of about $700 B_{\mathrm{S}}^{0} / \bar{B}_{\mathrm{S}}^{0} \rightarrow D_{\mathrm{S}}^{ \pm} K^{\mp}$ events is expected. This might allow for an initial measurement of $\sin \gamma$ with $2 \mathrm{fb}^{-1}$ in Run II.

$B^{0} \bar{B}^{0}$ and $B_{\mathrm{S}}^{0} \bar{B}_{\mathrm{S}}^{0}$ flavor oscillations measure the Cabibbo-Kobayashi-Maskawa matrix elements $\left|V_{t d}\right| /\left|V_{t s}\right|$. The recently approved detector upgrades play an important role in CDF's prospects for measuring $B_{\mathrm{S}}^{0}$ mixing. The additional inner layer of silicon improves the time resolution from $\sigma_{t}=0.060 \mathrm{ps}$ to $0.045 \mathrm{ps}$. This will be important if $\Delta m_{\mathrm{S}}$ is unexpectedly large. The time-of-flight system will enhance the effectiveness of $B$ flavor tagging, especially through same side tagging with kaons and opposite side 
kaon tagging, to a total $\varepsilon \mathcal{D}^{2} \sim 11.3 \%$. CDF expects a signal of 15,000-23,000 fully reconstructed $B_{\mathrm{S}}^{0} \rightarrow D_{\mathrm{S}}^{-} \pi^{+}, D_{\mathrm{S}}^{-} \pi^{+} \pi^{-} \pi^{+}$events from the two-track hadronic trigger in $2 \mathrm{fb}^{-1}$. For $20,000 B_{\mathrm{S}}^{0}$ events, a $5 \sigma$ measurement of $\Delta m_{\mathrm{S}}$ will be possible at CDF for $\Delta m_{\mathrm{S}}$ values up to $40 \mathrm{ps}^{-1}$. The current limit on $\Delta m_{\mathrm{S}}$ is $14.3 \mathrm{ps}^{-1}$ [7] at $95 \%$ C.L. It is noteworthy that physics with $B_{\mathrm{S}}^{0}$ mesons will be unique to the Tevatron until the turn-on of the LHC in 2006.

\section{Conclusions}

The CDF and DØ detector upgrades are well under way with data taking starting in March 2001. There are excellent prospects for $B$ physics in Run II, allowing a measurement of $\sin 2 \beta$ with an uncertainty of 0.07 . A discovery of $B_{\mathrm{S}}^{0}$ mixing is possible for $\Delta m_{\mathrm{S}}$ values up to $40 \mathrm{ps}^{-1}$. The extension of Run II until 2006 will further increase the sensitivity and the $B$ physics potential at the Tevatron. CDF and $\mathrm{D} \varnothing$ are looking forward to joining the party with the $B$ factories.

\section{References}

[1] C. P. Jessop et al. [CLEO Collaboration], Phys. Rev. Lett. 79, 4533 (1997).

[2] F. Abe et al. [CDF Collaboration], Phys. Rev. D59, 032001 (1999).

[3] T. Affolder et al. [CDF Collaboration], Phys. Rev. D61, 072005 (2000).

[4] F. Abe et al. [CDF Collaboration], Phys. Rev. Lett. 80, 2057 (1998).

[5] F. Abe et al. [CDF Collaboration], Phys. Rev. D60, 072003 (1999).

[6] Ron Poling, these proceedings.

[7] Guy Blaylock, these proceedings.

\section{Discussion}

Michail Danilov (ITEP, Moscow): What is the sensitivity of the D $\varnothing$ experiment for $B$ physics studies in Run II?

Paulini: As mentioned in my presentation, the prospects for $\mathrm{D} \varnothing$ are similar to the ones at CDF. DØ expects about $8500 J / \psi K_{S}^{0}$ events with $J / \psi \rightarrow \mu^{+} \mu^{-}$but they plan to also trigger on $e^{+} e^{-}$pairs resulting in additional $6500 \mathrm{~J} / \psi K_{S}^{0}$ events. From a 
time-dependent analysis, $\mathrm{D} \varnothing$ expects to measure $\sin 2 \beta$ with an uncertainty of 0.07 . In addition, an impact parameter trigger project has recently been approved allowing $\mathrm{D} \varnothing$ to detect $B \rightarrow \pi \pi$ events and also to explore $B_{\mathrm{S}}^{0} \bar{B}_{\mathrm{S}}^{0}$ mixing. 